\title{
Familial correlates of unmet need for contraception among currently married women: a comparative study of three Sub-Saharan African Countries
}

\author{
Eunice Ntwala S Imasiku \\ Department of Geography \& Environmental Studies, \\ University of Zambia, Zambia \\ eimasiku@yahoo.com
}

\begin{abstract}
Using data from the 2010 Malawi, 2013-14 Togo and 201I Uganda DHS, the study explored the relationship between unmet need for contraception (unmet need) and family factors. The analysis was based on data for 15,528 currently married women aged 15-49 in Malawi, 6,282 in Togo and 5,417 in Uganda. Multinomial regression models were used to analyse the data. Results show that Malawian and Togolese women in female headed households have a higher risk $(p<0.00 \mathrm{I})$ of having unmet need for spacing over no unmet need. Spousal agreement on fertility desire significantly reduced unmet need among Malawian women. Therefore family factors to some extent do influence unmet need in these countries. The findings indicate the importance of coming up with strategies which will encourage spousal communication in order to aid males and females to be equal partners in family planning.
\end{abstract}

Keywords: currently married women, Malawi, Togo, Uganda, unmet need for contraception.

\section{Introduction}

Despite the global commitment to family planning, the level of unmet need for contraception in developing countries still remains high. More than 200 women (I7\%) of reproductive age in these countries have unmet need for contraception (Singh and Darroch 2012). These women do not want to have any more children or want to wait for at least two years before having another child but are not using any contraceptives. Stover and Ross (2010); Kent (20I0); Ahmed, Li, Liu et al. (20I2) established that non-use of contraceptives is associated with adverse reproductive outcomes such as maternal mortality and infant and child mortality. These adverse reproductive health outcomes pose a serious public health concern.

Whereas a large amount of existing studies have established that unmet need for contraception is influenced by various socio-economic and demographic factors, studies addressing the influence of family factors on unmet need for contraception are lacking. In most cases only household wealth is included. However, there exists a big number of empirical works showing the influence of family factors on other reproductive health issues. Family factors play a significant role in one's life because the family is the closest tie that individuals have. Family factors are classified into three categories namely the contextual and structural features of families; family processes, relationships and practices; and biologic or hereditary influences (Miller, Benson and Galbraith 2001; Miller 2002). However, the last class does not apply to the current study. Contextual and structural features include couples' education, type of union and sex composition of children among others whereas family processes, relationships and practices include inter-spousal communication and agreement.

Many studies have assessed the effect of family structure on various reproductive health issues (Peres, Rutherford, Borgesa, et al. 2008; Stark, Tan, Muldoon et al. 2015). Concerning contraceptive use, Makatjane (1997) found that women in male-headed households were more likely to use modern contraceptives than their counterparts in femaleheaded households. In addition, studies such as Audu, Yahya, Geidama et al., (2008); Baschieri, Cleland, Floyd et al. (2013) reveal that women in polygamous marriages have lower odds of using contraceptives than those in monogamous marriages.

Inter-spousal communication and agreement are said to be significant elements for the proper functioning of the family in that couples who communicate are able to share information, ideas, and feelings as well as make decisions concerning important issues such contraceptive use (Hajason, Piña and Raveloharimisy 2013). Research has shown a significant association between inter-spousal communication and contraceptive use. For example Islam, Padmadas, and Smith (2010), established that inter-spousal communication was high among couples who used contraceptives. Another study indicated that inter-spousal communication was 1.9 times and 2.7 times likely to increase the odds of using modern 
and traditional contraceptives, respectively (Isam, Alam and Hasan 2014).

Therefore, this study's aim was to determine the family factors associated with unmet need for contraception among currently married women in Malawi, Togo and Uganda.

\section{Context}

Malawi, Togo and Uganda were chosen because the recent Demographic and Health Surveys of the three countries reveal that they have high levels of unmet need for contraception. The levels are 26.2\%, 33.6\% and $34.3 \%$ for Malawi, Togo and Uganda, respectively. Each of these countries has the highest level in the sub-region it belongs to. With this similarity, the study intends to find out if there are differences or not in the manner in which family factors influence unmet need for contraception among currently married women in the three countries.

\section{Data and methods}

The study utilised cross-sectional data from the 2010 Malawi Demographic and Health Survey (DHS), 2013-2014 Togo DHS and 20II Uganda DHS. The surveys are nationally representative and populationbased. In all the three countries, the primary sampling unit was regarded as a cluster which was defined on the basis of enumeration areas (EAs). The samples for all the surveys were selected using stratified twostage cluster design. EAs were the sampling units for the first stage whereas households were the second stage of sampling. Representative samples of 27,345 ; 9,549 and 10,086 households were selected for the surveys in Malawi, Togo and Uganda, respectively. The surveys collected information on demographic and health indicators from 23,020; 9,480 and 8,674 women aged 15-49 years in Malawi, Togo and Uganda, respectively. For more details on the methods refer to MS/Togo and ICF International (20I5), National Statistical Office (NSO) and ICF Macro (20II) and Uganda Bureau of Statistics (UBOS) and ICF International Inc. (20I2). Analysis for this study is based on data for currently married women aged 15-49 years. They were 15,528 of them in Malawi, 6,282 in Togo and 5,4I 7 in Uganda.

\section{Variables}

The outcome variable is unmet need for contraception. This refers to the non-use of contraception by fecund women who do not want to be pregnant, do not want any more children (unmet need for limiting) or want to wait for at least two years before having another child (unmet need for spacing). Included among these are pregnant or postpartum amenorrheic women whose pregnancy or current births are unwanted or mistimed. Those who are not using contraception and report that they are not sure whether they want another child or who want another child but are not sure when to have the birth are also considered to have unmet need for spacing. The variable was categorized as unmet need or contraception (no unmet need, unmet need for spacing and unmet need for limiting).

Family factors were the explanatory variables. These included type of marital union, sex of household head, sex composition of living children, household wealth, spousal age difference, number of children ever born and spousal agreement on fertility desire.

Four control variables were included. These were age, education, place of residence and exposure to family planning messages on media.

\section{Statistical analysis}

Data was analysed at the univariate, bivariate and multivariate levels. The first level involved describing the demographic and socio-economic characteristics of the study sample. At the bivariate level, the Pearson chi square test was used to determine whether unmet need for contraception was significantly associated with each of the explanatory variables. The multivariate level involved the use of multinomial regression models to explore the relationship between unmet need for spacing and limiting and selected explanatory variables respectively. This was because the dependent variable was polychotomous (no unmet need, unmet need for spacing, and unmet need for limiting).

Three models were fitted per country. The first model included the familial factors only whereas the second model added on the four control factors. The final model consisted of just those factors which were significant in the stepwise regression.

The analysis accounted for the complex DHS survey design to come up with efficient regression coefficients and robust standard errors while controlling for intra-cluster correlation. Sampling weights were used to ensure the representativeness of the sample. Individual sampling weights were used. Measures of association between the outcome and explanatory variables were expressed as relative risk ratios (RRR) with $95 \%$ level of confidence intervals $(\mathrm{Cl})$ and significance (p-values).

\section{Results \\ Background profile of the sample}

Table I provides background characteristics for the study sample. The majority of the women in all countries were aged between 25 and 34 years. More than half of these women were in monogamous unions and male headed households. Most (40\%) of 
these women had more sons than daughters. A higher proportion of these women were between I5 years younger than their spouses. In Malawi more than half $(55.9 \%)$ of the women had the same desired number of children with their spouses whereas more than half of their Togo and Uganda counterparts had different desired number of children. There were more women belonging to rich households than poor ones in all the countries. Moreover, most of the women in Malawi and Uganda had primary education whereas most of the women in Togo had no education. The majority of the women in all the countries resided in rural areas.

Background profile of currently married women aged 15-49 in Malawi, Togo and Uganda

\begin{tabular}{|c|c|c|c|c|c|c|}
\hline Selected characteristics & \multicolumn{2}{|l|}{ Malawi } & \multicolumn{2}{|l|}{ Togo } & \multicolumn{2}{|l|}{ Uganda } \\
\hline \multicolumn{7}{|l|}{ Age } \\
\hline $15-24$ & 4,639 & 29.9 & 1,107 & 17.63 & 1,505 & 27.78 \\
\hline $25-34$ & 6,354 & 40.9 & 2,620 & 41.70 & 2,176 & 40.16 \\
\hline $35-49$ & 4,534 & 29.2 & 2,555 & 40.67 & 1,737 & 32.06 \\
\hline \multicolumn{7}{|l|}{ Type of marriage } \\
\hline Monogamous & 13,180 & 84.9 & 4,211 & 67.09 & 3,890 & 71.80 \\
\hline Polygynous & 2,348 & 15.1 & 2,065 & 32.91 & 1,528 & 28.20 \\
\hline \multicolumn{7}{|l|}{ Sex of head of house hold } \\
\hline Male & 13,456 & 86.7 & 5,283 & 84.10 & 4,487 & 82.82 \\
\hline Female & 2,072 & 13.3 & 999 & 15.90 & 931 & 17.18 \\
\hline \multicolumn{7}{|c|}{ Sex composition of living children } \\
\hline Same & 3,848 & 24.8 & 1,524 & 24.26 & 1,229 & 22.68 \\
\hline Fewer sons & 5,503 & 35.4 & 2,143 & 34.11 & 1,986 & 36.66 \\
\hline More sons & 6,177 & 39.8 & 2,615 & 41.63 & 2,203 & 40.66 \\
\hline \multicolumn{7}{|l|}{ Spousal age difference } \\
\hline 0 & 956 & 6.2 & 409 & 6.51 & 521 & 9.61 \\
\hline $1-5$ & 8,152 & 52.5 & 2,427 & 38.65 & 2,367 & 43.70 \\
\hline $6-10$ & 4,370 & 28.1 & 1,889 & 30.07 & 1,545 & 28.52 \\
\hline$>10$ & 2,050 & 13.2 & 1,556 & 24.77 & 985 & 18.17 \\
\hline \multicolumn{7}{|c|}{ Spousal agreement on fertility desire } \\
\hline No & 6,181 & 44.1 & 4,094 & 65.50 & 3,697 & 70.35 \\
\hline Yes & 7,830 & 55.9 & 2,156 & 34.50 & 1,559 & 29.65 \\
\hline \multicolumn{7}{|l|}{$C E B$} \\
\hline 0 & 773 & 4.98 & 350 & 5.57 & 302 & 5.58 \\
\hline $1-2$ & 4,921 & 31.69 & 2,145 & 34.15 & 1,356 & 25.04 \\
\hline $3-4$ & 4,499 & 28.97 & 1,861 & 29.63 & 1,334 & 24.63 \\
\hline $5+$ & 5,334 & 34.35 & 1,925 & 30.65 & 2,425 & 44.76 \\
\hline \multicolumn{7}{|l|}{ Wealth } \\
\hline Poor & 5,759 & 37.1 & 2,435 & 38.76 & 2,164 & 39.94 \\
\hline Middle & 3,307 & 21.3 & 1,203 & 19.16 & 1,042 & 19.23 \\
\hline Rich & 6,465 & 41.6 & 2,644 & 42.08 & 2,212 & 40.84 \\
\hline \multicolumn{7}{|l|}{ Highest education } \\
\hline No education & 2,826 & 18.2 & 2,544 & 40.50 & 878 & 16.20 \\
\hline Primary & 10,231 & 65.9 & 2,253 & 35.86 & 3,313 & 61.15 \\
\hline Secondary/Higher & 2,470 & 15.9 & 1,485 & 23.64 & 1,227 & 22.66 \\
\hline \multicolumn{7}{|l|}{ Residence } \\
\hline Rural & 12,841 & 82.7 & 3,839 & 61.12 & 4,526 & 83.53 \\
\hline Urban & 2,686 & 17.3 & 2,443 & 38.88 & 892 & 16.47 \\
\hline \multicolumn{7}{|c|}{$\begin{array}{l}\text { Exposure to family planning messages } \\
\text { through media }\end{array}$} \\
\hline No & 5,757 & 37.2 & 4,984 & 79.40 & 1,370 & 25.30 \\
\hline Yes & 9,756 & 62.8 & 1,293 & 20.60 & 4,046 & 74.70 \\
\hline
\end{tabular}

Differentials in unmet need for contraception among currently married women in Malawi, Togo and Uganda

The distribution of currently married women in Malawi, Togo and Uganda by their unmet need for contraception status is presented in Table 2. The highest proportion of women with unmet need for spacing in all the countries were those aged 25-34 whereas for unmet need for limiting it was those aged 35-49. In all countries women in monogamous 
unions and male headed households had higher contraception. The percentage of women with unmet need for spacing in Malawi was highest among those with fewer sons whereas in Togo and Uganda it was among those with more sons. Malawian women who had the same desired number of children with their spouses had a higher percentage of unmet need for spacing whereas the opposite was the case for their Togo and Ugandan counterparts. In percentages of all components of unmet need for all countries, the majority of the women with unmet need for contraception belonged to poor households. Additionally, more than half of the women with unmet need for contraception in Malawi and Uganda had primary education whereas in Togo it was those with no education. A higher proportion of the women in all countries resided in rural areas.

Table 2: Distribution of currently married women aged I5-49 in Malawi, Togo and Uganda according to their unmet need for contraception status

\begin{tabular}{|c|c|c|c|c|c|c|c|c|c|}
\hline & & & Malawi & & & Togo & & & Uganda \\
\hline Selected characteristics & $\begin{array}{l}\text { Sp } \\
\%\end{array}$ & $\operatorname{Lim} \%$ & P-value & $\begin{array}{l}\text { Sp } \\
\%\end{array}$ & $\begin{array}{l}\operatorname{Lim} \\
\%\end{array}$ & P-value & $\begin{array}{l}\text { Sp } \\
\%\end{array}$ & $\operatorname{Lim}_{\%}$ & P-value \\
\hline Age & & & 0.000 & & & 0.000 & & & 0.000 \\
\hline $15-24$ & 43.6 & 16.2 & & 30.5 & 2.7 & & 41.67 & 5.07 & \\
\hline $25-34$ & 45.1 & 36.7 & & 51.3 & 26.4 & & 46.38 & 33.77 & \\
\hline $35-49$ & 11.3 & 47.1 & & 18.2 & 70.9 & & 11.96 & 61.16 & \\
\hline Type of marriage & & & 0.0081 & & & 0.0039 & & & 0.010 \\
\hline Monogamous & 85.4 & 81.0 & & 70.9 & 63.3 & & 73.46 & 67.68 & \\
\hline Polygynous & 14.6 & 19.0 & & 29.1 & 36.7 & & 26.54 & 32.32 & \\
\hline Sex of head of house hold & & & 0.0000 & & & 0.1721 & & & 0.044 \\
\hline Male & 80.0 & 85.6 & & 83.7 & 81.7 & & 83.61 & 80.29 & \\
\hline Female & 20.0 & 14.4 & & 16.3 & 18.3 & & 16.39 & 19.71 & \\
\hline $\begin{array}{l}\text { Sex composition of living } \\
\text { children }\end{array}$ & & & 0.0000 & & & 0.0015 & & & 0.0000 \\
\hline Same & 25.6 & 21.7 & & 23.0 & 19.4 & & 19.84 & 16.09 & \\
\hline Fewer sons & 38.2 & 36.8 & & 36.1 & 33.0 & & 39.86 & 35.65 & \\
\hline More sons & 36.2 & 41.6 & & 40.9 & 47.7 & & 40.31 & 48.26 & \\
\hline Spousal age difference & & & 0.0903 & & & 0.3056 & & & 0.002 \\
\hline 0 & 6.2 & 7.7 & & 5.7 & 7.4 & & 8.15 & 11.01 & \\
\hline $1-5$ & 54.0 & 49.3 & & 39.7 & 35.0 & & 45.29 & 38.70 & \\
\hline $6-10$ & 27.6 & 28.0 & & 30.2 & 29.5 & & 28.80 & 27.97 & \\
\hline$>10$ & 12.2 & 15.0 & & 24.5 & 28.1 & & 17.75 & 22.32 & \\
\hline $\begin{array}{l}\text { Spousal agreement on fertility } \\
\text { desire }\end{array}$ & & & 0.000 & & & 0.578 & & & 0.000 \\
\hline No & 44.3 & 52.5 & & 66.1 & 69.4 & & 68.66 & 80.14 & \\
\hline Yes & 55.7 & 47.5 & & 33.9 & 30.6 & & 31.34 & 19.86 & \\
\hline CEB & & & 0.000 & & & 0.000 & & & 0.000 \\
\hline 0 & 5.3 & 2.3 & & 2.9 & 0.1 & & 3.80 & 0.15 & \\
\hline $\mathrm{I}-2$ & 42.5 & 16.4 & & 47.7 & 8.5 & & 33.70 & 3.91 & \\
\hline 3-4 & 33.4 & 27.6 & & 30.6 & 32.9 & & 30.71 & 11.74 & \\
\hline $5+$ & 18.8 & 53.7 & & 18.8 & 58.5 & & 31.79 & 84.20 & \\
\hline Wealth & & & 0.000 & & & 0.149 & & & 0.000 \\
\hline Poor & 41.8 & 40.3 & & 41.8 & 35.0 & & 46.92 & 48.26 & \\
\hline Middle & 24.6 & 19.3 & & 20.2 & 18.2 & & 17.66 & $|8.4|$ & \\
\hline Rich & 33.6 & 40.4 & & 37.9 & 46.8 & & 35.42 & 33.33 & \\
\hline Highest education & & & 0.000 & & & 0.000 & & & 0.000 \\
\hline No education & 14.5 & 24.9 & & 38.6 & 48.0 & & 11.96 & 25.65 & \\
\hline Primary & 69.9 & 65.6 & & 36.0 & 35.4 & & 66.85 & 62.75 & \\
\hline Secondary/Higher & 15.7 & 9.5 & & 25.4 & 16.6 & & 21.20 & 11.59 & \\
\hline Residence & & & 0.0110 & & & 0.0004 & & & 0.000 \\
\hline Rural & 86.7 & 83.2 & & 65.3 & 55.4 & & 80.07 & 84.49 & \\
\hline Urban & 13.3 & 16.8 & & 34.7 & 44.6 & & 19.93 & $15.5 \mid$ & \\
\hline $\begin{array}{l}\text { Exposure to family planning } \\
\text { messages through media }\end{array}$ & & & 0.0004 & & & 0.0001 & & & 0.013 \\
\hline No $\quad$ - n & 40.9 & 40.7 & & 65.3 & 55.4 & 0.0004 & 26.00 & 32.46 & \\
\hline Yes & 59.1 & 59.3 & & 34.7 & 44.6 & & 74.00 & 67.54 & \\
\hline
\end{tabular}


Familial factors associated with unmet need for contraception among currently married women in Malawi, Togo and Uganda

Results of the multinomial logistic regression analysis presented in Tables 3, 4 and 5 reveal that when family factors are unadjusted, the relative risk ratio for Togolese women in polygynous unions to have unmet need for spacing over no unmet need was $0.78(p<0.01)$. Malawian women in female headed households were $80 \%(p<0.00 \mathrm{I})$ more likely to have unmet need for spacing over no unmet need. Sex composition of living children was significantly associated with unmet need for spacing in Uganda. Togolese women whose fertility desire was the same as their spouses were $14 \%(p<0.05)$ less likely to have unmet need for spacing over no unmet need. The number of children ever born (I-2 and 3-4) was positively associated with unmet need for spacing over no unmet need in Togo and Uganda. Women belonging to rich households in all countries had reduced risks of having unmet need for spacing over no unmet need.

After including the control variables, the relationship between type of union and unmet need for spacing among women in Togo and sex composition of living children ceased being significant. The association between sex composition of living children and unmet need for spacing among Ugandan women and the relationship between spousal agreement on fertility desire among women in Togo also lost their significance. The association between number of children ever born (I-2 and 3-4) and unmet need for spacing among Malawian women became significant. Among the added variables, age was inversely associated with unmet need for spacing among women in all countries. Additionally, women residing in urban areas in Uganda were $28 \%$ $(p<0.05)$ less likely to have unmet need for spacing over no unmet need.

Table 3: Relative risk ratios from multinomial logistic regressions predicting unmet need for spacing and limiting among currently married women, MDHS 2010

\begin{tabular}{|c|c|c|c|}
\hline \multicolumn{4}{|l|}{ Base outcome: Met need } \\
\hline Unmet need for spacing & Model I & Model 2 & Model 3 \\
\hline Selected characteristics & RRR (Cl 95\%) & RRR (Cl 95\%) & RRR (Cl 95\%) \\
\hline \multicolumn{4}{|l|}{ Age } \\
\hline $15-24$ & & 1.00 & 1.00 \\
\hline $25-34$ & & $0.77(0.65-0.92)^{* * *}$ & $0.78(0.65-0.92)^{* *}$ \\
\hline $35-49$ & & $0.39(0.30-0.50)^{* * * *}$ & $0.39(0.31-0.50)^{* * * *}$ \\
\hline \multicolumn{4}{|l|}{ Type of marriage } \\
\hline Monogamous & 1.00 & 1.00 & \\
\hline Polygynous & $1.02(0.85-1.23)$ & $\mathrm{I} .08(0.90-\mathrm{I} .30)$ & \\
\hline \multicolumn{4}{|l|}{ Sex of head of house hold } \\
\hline Male & 1.00 & 1.00 & 1.00 \\
\hline Female & $1.80(1.53-2.13)^{* * * *}$ & $\mathrm{I} .79(\mathrm{I} .52-2 . \mathrm{II})^{* * * *}$ & $1.79(\mathrm{I} .52-2.1 \mathrm{I})^{* * * *}$ \\
\hline \multicolumn{4}{|c|}{ Sex composition of living children } \\
\hline Same & 1.00 & 1.00 & 1.00 \\
\hline Fewer sons & $1.13(0.95-1.35)$ & $\mathrm{I} .09(0.92-\mathrm{I} .3 \mathrm{I})$ & $\mathrm{I} .10(0.92-\mathrm{I} .3 \mathrm{I})$ \\
\hline More sons & $0.95(0.79-|| 3)$. & $0.93(0.78-\mathrm{I} .12)$ & $0.94(0.78-\mathrm{I} .12)$ \\
\hline \multicolumn{4}{|c|}{ Spousal age difference (man's age - woman's) } \\
\hline 0 & 1.00 & 1.00 & \\
\hline $\mathrm{I}-5$ & $0.91(0.68-1.20)$ & $0.80(0.60-\mathrm{I} .06)$ & \\
\hline $6-10$ & $0.89(0.67-1.20)$ & $0.79(0.59-1.06)$ & \\
\hline$>10$ & $0.93(0.67-1.29)$ & $0.85(0.62-I .19)$ & \\
\hline \multicolumn{4}{|c|}{ Spousal agreement on fertility desire } \\
\hline No & 1.00 & 1.00 & 1.00 \\
\hline Yes & $0.89(0.79-1.02)$ & $0.88(0.77-\mathrm{I} .00)$ & $0.87(0.77-\mathrm{I} .00)^{*}$ \\
\hline \multicolumn{4}{|l|}{$C E B$} \\
\hline 0 & 1.00 & 1.00 & 1.00 \\
\hline $\mathrm{I}-2$ & $1.34(0.97-1.85)$ & $\mathrm{I} .4 \mathrm{I}(\mathrm{I} .02-\mathrm{I} .94)^{*}$ & $1.42(1.03-1.97)^{*}$ \\
\hline $3-4$ & $1.31(0.94-1.82)$ & $1.59(1.12-2.26)^{* *}$ & $1.61(1.14-2.29)^{* *}$ \\
\hline $5+$ & $0.82(0.58-I .15)$ & $1.38(0.95-2.01)$ & $1.40(0.96-2.03)$ \\
\hline \multicolumn{4}{|l|}{ Wealth } \\
\hline Poor & 1.00 & 1.00 & \\
\hline Middle & I.04 (0.89-I.22) & $\mathrm{I} .09(0.93-\mathrm{I} .28)$ & \\
\hline Rich & $0.7 \mathrm{I}(0.6 \mathrm{I}-0.83)^{* * * *}$ & $0.84(0.7 \mid-0.99)^{*}$ & \\
\hline \multicolumn{4}{|l|}{ Highest education } \\
\hline No education & & 1.00 & 1.00 \\
\hline Primary & & I.0I (0.84-I.22) & $0.98(0.82-I .18)$ \\
\hline Secondary/Higher & & $0.93(0.72-I .2 I)$ & $0.86(0.67-I .10)$ \\
\hline
\end{tabular}


African Population Studies Vol 3I, No I, (Supp. 2), 2017

\begin{tabular}{|c|c|c|c|}
\hline \multicolumn{4}{|l|}{ Residence } \\
\hline Rural & & 1.00 & 1.00 \\
\hline Urban & & $0.85(0.67-1.09)$ & $0.77(0.6 \mathrm{I}-0.97)^{*}$ \\
\hline \multicolumn{4}{|c|}{$\begin{array}{l}\text { Exposure to family planning messages through } \\
\text { media }\end{array}$} \\
\hline No & & 1.00 & 1.00 \\
\hline Yes & & $0.89(0.77-I .02)$ & $0.87(0.76-\mathrm{I}-00) *$ \\
\hline Unmet need for limiting & Model I & Model 2 & Model 3 \\
\hline Selected characteristics & RRR (Cl 95\%) & RRR (Cl 95\%) & RRR (Cl 95\%) \\
\hline \multicolumn{4}{|l|}{ Age } \\
\hline $15-24$ & & 1.00 & 1.00 \\
\hline $25-34$ & & $0.96(0.77-I .19)$ & 0.97 \\
\hline $35-49$ & & $1.60(1.25-2.05)^{* * * *}$ & $1.63 * * *$ \\
\hline \multicolumn{4}{|l|}{ Type of marriage } \\
\hline Monogamous & 1.00 & 1.00 & \\
\hline Polygynous & $\mathrm{I} .08(0.93-\mathrm{I} .27)$ & I.07 (0.9I-I.26) & \\
\hline \multicolumn{4}{|l|}{ Sex of head of house hold } \\
\hline Male & 1.00 & 1.00 & 1.00 \\
\hline Female & $1.35(1.13-1.60)^{* *}$ & $1.36(1.14-1.62)^{* *}$ & $1.37(1.15-1.63)^{* * * *}$ \\
\hline \multicolumn{4}{|c|}{ Sex composition of living children } \\
\hline Same & 1.00 & 1.00 & 1.00 \\
\hline Less sons & $0.91(0.77-1.08)$ & $0.92(0.78-I .10)$ & $0.92(0.77-1.09)$ \\
\hline More sons & $0.88(0.74-\mathrm{I} .04)$ & $0.87(0.74-\mathrm{I} .03)$ & $0.86(0.73-\mathrm{I} .03)$ \\
\hline \multicolumn{4}{|c|}{ Spousal age difference (man's age - woman's) } \\
\hline 0 & 1.00 & 1.00 & \\
\hline $\mathrm{I}-5$ & $0.86(0.68-1.09)$ & $0.93(0.74-1.18)$ & \\
\hline $6-10$ & $0.79(0.62-1.01)$ & $0.84(0.66-1.07)$ & \\
\hline$>10$ & $0.82(0.63-\mathrm{I} .07)$ & $0.85(0.65-\mathrm{I} . \mathrm{II})$ & \\
\hline \multicolumn{4}{|c|}{ Spousal agreement on fertility desire } \\
\hline No & 1.00 & 1.00 & 1.00 \\
\hline Yes & $0.78(0.69-0.89)$ **** & $0.81(0.72-0.92)^{* *}$ & $0.8 \mathrm{I}(0.7 \mid-0.9 \mathrm{I})^{* * *}$ \\
\hline \multicolumn{4}{|l|}{ CEB } \\
\hline 0 & 1.00 & 1.00 & 1.00 \\
\hline $\mathrm{I}-2$ & $1.37(0.90-2.10)$ & $\mathrm{I} .4 \mathrm{I}(0.93-2.16)$ & $1.42(0.93-2.16)$ \\
\hline $3-4$ & $2.92(1.92-4.45)^{* * * *}$ & $2.89(1.87-4.45)^{* * * *}$ & $2.87(1.87-4.42)^{* * * *}$ \\
\hline $5+$ & $6.37(4.21-9.64)^{* * * *}$ & $4.88(3.15-7.56)^{* * * *}$ & $4.83(3.13-7.48)^{* * * *}$ \\
\hline \multicolumn{4}{|l|}{ Wealth } \\
\hline Poor & 1.00 & 1.00 & \\
\hline Middle & $0.89(0.76-1.04)$ & $0.90(0.77-1.05)$ & \\
\hline Rich & $1.06(0.93-I .22)$ & $1.00(0.87-1.17)$ & \\
\hline \multicolumn{4}{|l|}{ Highest education } \\
\hline No education & & 1.00 & 1.00 \\
\hline Primary & & I.0I (0.87-I.I8) & $\mathrm{I} .02(0.88-\mathrm{I} .18)$ \\
\hline Secondary/Higher & & $0.79(0.60-1.04)$ & $0.81(0.61-1.05)$ \\
\hline \multicolumn{4}{|l|}{ Residence } \\
\hline Rural & & 1.00 & 1.00 \\
\hline Urban & & $1.40(1.13-1.73)^{* * *}$ & $\mathrm{I} .4 \mathrm{I}(\mathrm{I} . \mathrm{I} 4-\mathrm{I} .73)^{* * *}$ \\
\hline \multicolumn{4}{|c|}{$\begin{array}{l}\text { Exposure to family planning messages through } \\
\text { media }\end{array}$} \\
\hline No & & 1.00 & 1.00 \\
\hline Yes & & $0.88(0.77-1.00)$ & $0.88(0.77-1.00)^{*}$ \\
\hline
\end{tabular}

Table 4: Relative risk ratios from multinomial logistic regressions predicting unmet need for spacing and limiting among currently married women, TDHS 2013-14

\begin{tabular}{|l|l|l|l|}
\hline Base outcome: No unmet need & & & \\
\hline Unmet need for spacing & Model I & Model 2 & Model 3 \\
\hline Selected characteristics & RRR (CI 95\%) & RRR (Cl 95\%) & RRR (CI 95\%) \\
\hline Age & & & \\
\hline I5-24 & & 1.00 & 1.00 \\
\hline $25-34$ & & $0.57(0.47-0.69)^{* * * *}$ & $0.57(0.47-0.69)^{* * * *}$ \\
\hline $35-49$ & & $0.19(0.15-0.25)^{* * * *}$ & $0.19(0.15-0.24)^{* * * *}$ \\
\hline Type of marriage & & & \\
\hline Monogamous & $\mathrm{I} .00$ & $\mathrm{I} .00$ & \\
\hline Polygynous & $0.78(0.67-0.91)^{* *}$ & $0.86(0.73-1.01)$ & \\
\hline
\end{tabular}


African Population Studies Vol. 3I, No. I, (Supp. 2), 2017

\begin{tabular}{|c|c|c|c|}
\hline \multicolumn{4}{|l|}{ Sex of head of house hold } \\
\hline Male & 1.00 & 1.00 & 1.00 \\
\hline Female & $1.11(0.91-1.35)$ & $1.26(1.03-1.54)^{*}$ & $1.22(1.00-1.49) * * *$ \\
\hline \multicolumn{4}{|c|}{ Sex composition of living children } \\
\hline Same & 1.00 & 1.00 & 1.00 \\
\hline Fewer sons & $1.04(0.85-1.26)$ & $0.93(0.76-1.14)$ & $0.93(0.76-I .13)$ \\
\hline More sons & $0.99(0.82-1.20)$ & $0.9 \mathrm{I}(0.75-\mathrm{I} . \mathrm{II})$ & $0.9 \mathrm{I}(0.75-\mathrm{I} . \mathrm{II})$ \\
\hline \multicolumn{4}{|c|}{ Spousal age difference (man's age - woman's) } \\
\hline 0 & 1.00 & 1.00 & \\
\hline $\mathrm{I}-5$ & $1.09(0.79-1.50)$ & $0.87(0.62-1.22)$ & \\
\hline $6-10$ & $1.16(0.83-1.61)$ & $0.88(0.62-1.23)$ & \\
\hline$>10$ & $1.21(0.86-1.69)$ & $0.93(0.66-1.32)$ & \\
\hline \multicolumn{4}{|c|}{ Spousal agreement on fertility desire } \\
\hline No & 1.00 & 1.00 & \\
\hline Yes & $0.86(0.75-1.00)^{*}$ & $0.86(0.74-1.00)$ & \\
\hline \multicolumn{4}{|l|}{ CEB } \\
\hline 0 & 1.00 & 1.00 & 1.00 \\
\hline $\mathrm{I}-2$ & $3.67(2.45-5.49) * * * *$ & $4.36(2.91-6.53)^{* * * *}$ & $4.33(2.90-6.48) * * *$ \\
\hline $3-4$ & $2.76(1.83-4.17)^{* * * *}$ & $4.99(3.28-7.59)^{* * * *}$ & $4.94(3.24-7.52) * * * *$ \\
\hline $5+$ & $1.48(0.96-2.27)$ & $4.29(2.73-6.75) * * *$ & $4.32(2.75-6.79) * * *$ \\
\hline \multicolumn{4}{|l|}{ Wealth } \\
\hline Poor & 1.00 & 1.00 & 1.00 \\
\hline Middle & $0.85(0.70-1.02)$ & $0.90(0.74-1.09)$ & $0.90(0.74-I .09)$ \\
\hline Rich & $0.62(0.53-0.73)^{* * * *}$ & $0.81(0.59-1.09)$ & $0.80(0.59-1.07)$ \\
\hline \multicolumn{4}{|l|}{ Highest education } \\
\hline No education & & 1.00 & \\
\hline Primary & & $0.93(0.79-1.10)$ & \\
\hline Secondary/Higher & & $0.94(0.77-1.16)$ & \\
\hline \multicolumn{4}{|l|}{ Residence } \\
\hline Rural & & 1.00 & 1.00 \\
\hline Urban & & $0.95(0.70-1.27)$ & $0.95(0.71-1.28)$ \\
\hline \multicolumn{4}{|c|}{$\begin{array}{l}\text { Exposure to family planning messages through } \\
\text { media }\end{array}$} \\
\hline No & & 1.00 & \\
\hline Yes & & $0.97(0.82-1.16)$ & \\
\hline Unmet need for limiting & Model I & Model 2 & Model 3 \\
\hline Selected characteristics & RRR (Cl 95\%) & RRR (Cl 95\%) & RRR (Cl 95\%) \\
\hline \multicolumn{4}{|l|}{ Age } \\
\hline $15-24$ & & 1.00 & 1.00 \\
\hline $25-34$ & & $1.29(0.75-2.20)$ & $1.28(0.74-2.19)$ \\
\hline $35-49$ & & $2.05(1.19-3.54)^{*}$ & $1.99(1.15-3.43)^{*}$ \\
\hline \multicolumn{4}{|l|}{ Type of marriage } \\
\hline Monogamous & 1.00 & 1.00 & \\
\hline Polygynous & $0.87(0.71-1.06)$ & $0.84(0.68-1.03)$ & \\
\hline \multicolumn{4}{|l|}{ Sex of head of house hold } \\
\hline Male & 1.00 & 1.00 & 1.00 \\
\hline Female & $1.60(1.24-2.06)^{* * * *}$ & $1.49(1.15-1.94)^{* * *}$ & $1.44(1.11-1.86)^{* * * *}$ \\
\hline \multicolumn{4}{|c|}{ Sex composition of living children } \\
\hline Same & 1.00 & 1.00 & 1.00 \\
\hline Less sons & $0.69(0.53-0.89)^{* * *}$ & $0.69(0.53-0.90)^{* * *}$ & $0.70(0.54-0.91)^{* * *}$ \\
\hline More sons & $0.82(0.64-1.05)$ & $0.81(0.63-1.03)$ & $0.82(0.64-1.05)$ \\
\hline \multicolumn{4}{|c|}{ Spousal age difference (man's age - woman's) } \\
\hline 0 & 1.00 & 1.00 & \\
\hline I-5 & $0.92(0.64-1.33)$ & $0.97(0.67-1.40)$ & \\
\hline $6-10$ & $0.91(0.63-1.32)$ & $0.98(0.68-I .4 I)$ & \\
\hline$>10$ & $1.11(0.76-1.63)$ & $1.18(0.81-1.73)$ & \\
\hline \multicolumn{4}{|c|}{ Spousal agreement on fertility desire } \\
\hline No & 1.00 & 1.00 & \\
\hline Yes & $0.96(0.78-1.17)$ & $0.96(0.78-1.18)$ & \\
\hline CEB & & & \\
\hline 0 & 1.00 & 1.00 & 1.00 \\
\hline $\mathrm{I}-2$ & $22(3.00-163.23) * *$ & $21(2.88-154 . .42) * *$ & $20(2.79-149.14)^{* *}$ \\
\hline 3-4 & $126(17.33-923.35)^{* * * *}$ & $103(14.16-742.00) * * *$ & $97(\mid 3.42-696.51)^{* * * *}$ \\
\hline $5+$ & $297(40.56-2180.74) * * *$ & $206(28.21-1503.36) * * *$ & $197(27.09-\mid 425.39) * * *$ \\
\hline Wealth & & & \\
\hline
\end{tabular}


African Population Studies Vol 3I, No I, (Supp. 2), 2017

\begin{tabular}{|l|l|l|l|}
\hline Poor & $\mathrm{I} .00$ & $\mathrm{I} .00$ & $\mathrm{I} .00$ \\
\hline Middle & $\mathrm{I} .27(0.99-\mathrm{I} .64)$ & $\mathrm{I} .26(0.98-\mathrm{I} .63)$ & $\mathrm{I} .25(0.97-\mathrm{I} .6 \mathrm{I})$ \\
\hline Rich & $2.52(2.03-3 . \mathrm{I})^{* * *}$ & $\mathrm{I} .99(\mathrm{I} .34-2.97)^{* *}$ & $\mathrm{I} .96(\mathrm{I} .33-2.89)^{*}$ \\
\hline Highest education & & & \\
\hline No education & & $\mathrm{I} .00$ & \\
\hline Primary & & $0.95(0.77-\mathrm{I} . \mathrm{I} 8)$ & \\
\hline Secondary/Higher & & $\mathrm{I} .00(0.75-\mathrm{I} .35)$ & \\
\hline Residence & & & \\
\hline Rural & & $\mathrm{I} .00$ & $\mathrm{I} .00$ \\
\hline Urban & & $\mathrm{I} .29(0.89-\mathrm{I} .86)$ & $\mathrm{I} .29(0.90-\mathrm{I} .86)$ \\
\hline $\begin{array}{l}\text { Exposure to family planning messages through } \\
\text { media }\end{array}$ & & & \\
\hline No & & $\mathrm{I} .00$ & \\
\hline Yes & & $0.92(0.73-\mathrm{I} .16)$ & \\
\hline
\end{tabular}

${ }^{\mathrm{R}}=$ Reference category, ${ }^{*}=\mathrm{p}<0.05,{ }^{*}=\mathrm{p}<0.0 \mathrm{I},{ }^{*} * *=\mathrm{p}<0.00 \mathrm{I}$

In the second model, the pattern of association between the familial factors and unmet need for spacing in all the countries was the same as that in the previous model except that in Malawi spousal agreement on fertility and place of residence became significantly associated with unmet need for spacing.

Tables 3, 4 and 5 also reveal the influences of familial factors on unmet need for limiting in Malawi, Togo and Uganda. The unadjusted effects of familial factors on unmet need for limiting in Malawi are as follows. Women in female headed households had higher risks ( $R R R=1.35, p<0.0 \mathrm{I})$ of having unmet need for limiting over no unmet need. Spousal agreement on fertility desire was negatively associated with unmet need for limiting ( $R R R=0.78$, $\mathrm{P}<0.000$ whereas the number of children born was positively associated with unmet need for limiting over no unmet need.

In Togo, Women in female headed households had higher risks ( $R R R=1.60, p<0.00 I)$ of having unmet need for limiting over no unmet need. Women with fewer sons than daughters were less likely to have unmet need for limiting over no unmet need. The number of children ever born was positively associated with unmet need for limiting over no unmet need whereas women belonging to rich households had higher risks ( $R R R=2.52$, $P<0.00 \mathrm{I}$ ) of unmet need for limiting over no unmet need.

The number of children ever born was positively associated with unmet need for limiting over no unmet need among Ugandan women. On the contrary, wealth was negatively associated with unmet need for limiting.

After controlling for age, education, place of residence and exposure to family planning message on media, the pattern of association between the familial factors and unmet need for limiting in all the countries was the same as that in the previous model. Among the added variables, results for all countries reveal that the risk of having unmet need for limiting over no unmet need was higher among women aged 35-49 years. Additionally, Malawian women residing in urban areas were more likely $(R R R=I .40, P=0.01)$ to have unmet need for limiting over no unmet need.

Table 5: Relative risk ratios from multinomial logistic regressions predicting unmet need for spacing and limiting among currently married women, UDHS 20I I

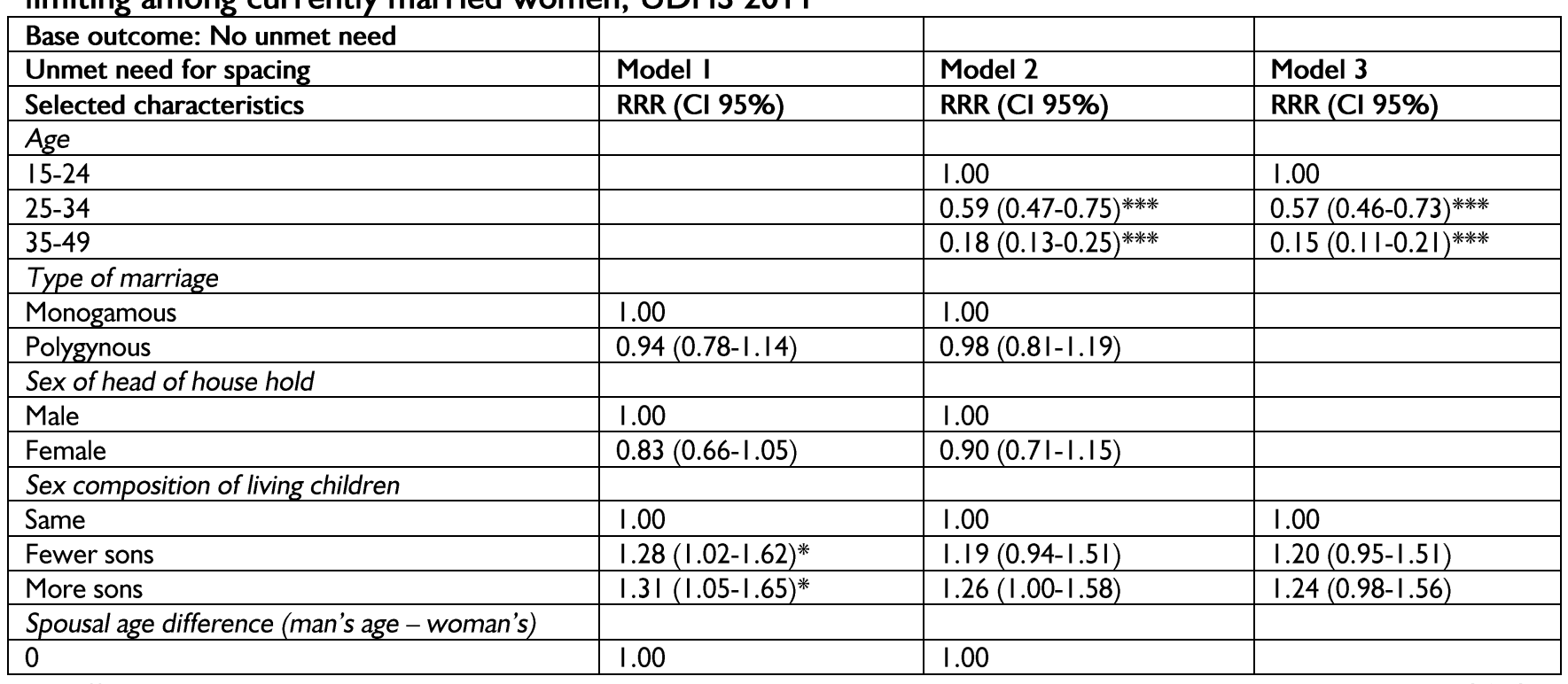




\begin{tabular}{|c|c|c|c|}
\hline $\mathrm{I}-5$ & $1.17(0.87-1.57)$ & $0.95(0.70-1.30)$ & \\
\hline $6-10$ & $1.20(0.88-1.63)$ & $0.96(0.69-1.33)$ & \\
\hline$>10$ & $1.29(0.92-1.81)$ & $1.06(0.75-I .5 I)$ & \\
\hline \multicolumn{4}{|c|}{ Spousal agreement on fertility desire } \\
\hline No & 1.00 & 1.00 & \\
\hline Yes & $1.01(0.85-1.20)$ & $0.97(0.82-1.16)$ & \\
\hline \multicolumn{4}{|l|}{ CEB } \\
\hline 0 & 1.00 & 1.00 & 1.00 \\
\hline $\mid-2$ & $1.95(1.20-3.16)^{* * *}$ & $2.26(1.39-3.67)^{* *}$ & $2.30(1.42-3.76)$ \\
\hline $3-4$ & $2.00(1.22-3.26)^{* * *}$ & $3.00(1.79-5.01)^{* * * *}$ & $3.05(1.82-5.10)$ \\
\hline $5+$ & $1.28(0.78-2.09)$ & $3.44(2.01-5.89) * * *$ & $3.39(1.97-5.82) * * *$ \\
\hline \multicolumn{4}{|l|}{ Wealth } \\
\hline Poor & 1.00 & 1.00 & \\
\hline Middle & $0.77(0.62-0.96)^{* * *}$ & $0.78(0.62-0.98)^{*}$ & \\
\hline Rich & $0.59(0.50-0.71)^{* * *}$ & $0.75(0.60-0.94)^{*}$ & \\
\hline \multicolumn{4}{|l|}{ Highest education } \\
\hline No education & & 1.00 & 1.00 \\
\hline Primary & & $1.50(1.16-1.96)^{* *}$ & $1.42(1.10-1.83)^{* * *}$ \\
\hline Secondary/Higher & & $1.19(0.85-1.67)$ & $1.05(078-1.43)$ \\
\hline \multicolumn{4}{|l|}{ Residence } \\
\hline Rural & & 1.00 & 1.00 \\
\hline Urban & & $0.72(0.56-0.93)^{*}$ & $0.65(0.52-0.82) * * *$ \\
\hline \multicolumn{4}{|c|}{$\begin{array}{l}\text { Exposure to family planning messages through } \\
\text { media }\end{array}$} \\
\hline No & & 1.00 & \\
\hline Yes & & $1.10(0.90-1.33)$ & \\
\hline Unmet need for limiting & Model I & Model 2 & Model 3 \\
\hline Selected characteristics & $\operatorname{RRR~(Cl~95\% )~}$ & RRR (Cl 95\%) & $\operatorname{RRR~(Cl~95\% )~}$ \\
\hline \multicolumn{4}{|l|}{ Age } \\
\hline $15-24$ & & 1.00 & 1.00 \\
\hline $25-34$ & & $1.46(0.86-2.48)$ & $1.39(0.82-2.35)$ \\
\hline $35-49$ & & $2.01(1.16-3.48)^{*}$ & $1.68(0.97-2.89)$ \\
\hline \multicolumn{4}{|l|}{ Type of marriage } \\
\hline Monogamous & 1.00 & 1.00 & \\
\hline Polygynous & $0.8 I(0.65-1.02)$ & $0.8 I(0.64-I .02)$ & \\
\hline \multicolumn{4}{|l|}{ Sex of head of house hold } \\
\hline Male & 1.00 & 1.00 & \\
\hline Female & $1.20(0.92-1.59)$ & $1.23(0.94-1.62)$ & \\
\hline \multicolumn{4}{|c|}{ Sex composition of living children } \\
\hline Same & 1.00 & 1.00 & 1.00 \\
\hline Less sons & $0.90(0.67-1.23)$ & $0.91(0.67-1.23)$ & $0.89(0.66-I .2 I)$ \\
\hline More sons & $1.07(0.80-\mathrm{I} .44)$ & $1.06(0.79-1.42)$ & $1.02(0.76-1.37)$ \\
\hline \multicolumn{4}{|c|}{ Spousal age difference (man's age - woman's) } \\
\hline 0 & 1.00 & 1.00 & \\
\hline $1-5$ & $0.98(0.69-1.39)$ & $1.02(0.72-1.44)$ & \\
\hline $6-10$ & $1.05(0.73-1.52)$ & $1.12(0.77-1.6 \mathrm{I})$ & \\
\hline$>10$ & $1.22(0.84-1.79)$ & $1.28(0.87-1.88)$ & \\
\hline \multicolumn{4}{|c|}{ Spousal agreement on fertility desire } \\
\hline No & 1.00 & 1.00 & \\
\hline Yes & $0.83(0.64-1.07)$ & $0.85(0.66-1.10)$ & \\
\hline \multicolumn{4}{|l|}{ CEB } \\
\hline 0 & 1.00 & 1.00 & 1.00 \\
\hline $\mathrm{I}-2$ & $5.27(0.69-40.23)$ & $5.12(0.68-38.62)$ & $5.30(0.70-39.96)$ \\
\hline $3-4$ & $15.58(2.10-115.40)^{* *}$ & $12.35(1.66-91.89)^{*}$ & $12.72(1.71-94.50)^{*}$ \\
\hline $5+$ & $79.38(10.89-578.72)^{* * * *}$ & $47.84(6.42-356.48) * * *$ & $47.97(6.46-356.28)^{* * * *}$ \\
\hline \multicolumn{4}{|l|}{ Wealth } \\
\hline Poor & 1.00 & 1.00 & \\
\hline Middle & $0.73(0.56-0.95)^{*}$ & $0.73(0.56-0.95)^{*}$ & \\
\hline Rich & $0.67(0.53-0.4)^{* * *}$ & $0.76(0.57-1.01)$ & \\
\hline \multicolumn{4}{|l|}{ Highest education } \\
\hline No education & & 1.00 & 1.00 \\
\hline Primary & & $1.20(0.94-1.53)$ & $1.03(0.81-1.30)$ \\
\hline Secondary/Higher & & $0.86(0.57-I .28)$ & $0.69(0.48-0.99)^{*}$ \\
\hline Residence & & & \\
\hline Rural & & 1.00 & 1.00 \\
\hline
\end{tabular}




\begin{tabular}{|l|l|l|l|}
\hline Urban & & $0.79(0.56-\mathrm{I} . \mathrm{I} 2)$ & $0.73(0.53-0.99)^{*}$ \\
\hline $\begin{array}{l}\text { Exposure to family planning messages through } \\
\text { media }\end{array}$ & & & \\
\hline No & & $\mathrm{I} .00$ & \\
\hline Yes & & $0.88(0.70-\mathrm{I} . \mathrm{II})$ & \\
${ }_{\mathrm{R}}=$ Reference category, $* \mathrm{p}<0.05, * *=\mathrm{p}<0.0 \mathrm{I}, * * *$ \\
\end{tabular}

In the final model, the only changes that occurred are that in Malawi and Uganda exposure to family planning messages through media and place of residence became significantly associated with unmet need for limiting.

\section{Discussion}

The study compared the familial correlates of unmet need for contraception among currently married women in Malawi, Togo and Uganda. After adjusting for control factors (age, educational attainment, place of residence and exposure to family planning messages on media) results of the study indicate that some of the familial factors are associated with unmet need for contraception in Malawi, Togo and Uganda. The major familial correlates of unmet need for spacing and limiting in Malawi are sex of the household head, spousal agreement on desired number of children and number of children ever born. In Togo the major familial correlates of unmet need for spacing and limiting are sex of the household head and number of children ever born. As for Uganda it is only the number of children ever born.

Findings of this study reveal that the number of children ever born was positively associated with unmet need for contraception in all the three countries. In other words women with high fertility had higher risks of having unmet need for contraception. This suggests that there is a culture of having many children in these countries and as such women are less likely to use contraceptives.

Malawian and Togolese women in female headed households are more likely to have unmet need for contraception than those in male headed households. This is consistent with earlier findings (Oginni, Ahonsi and Adebajo (20I5). This suggests that such women have a lower likelihood of using contraceptives. The reason for the lower likelihood of using contraceptives could be poverty. Women from poor households lack access to better family planning. Actually, several studies attest to the fact that that female headed households tend to be poorer than male headed ones (Horrell and Krishnan 2007; Rahman, Matsui and Ikemoto 2013). It appears that males in these two countries have more control of resources than the females.

Another finding worth noting is the significant relationship between spousal agreement on fertility desire and unmet need for contraception. Malawian women whose fertility desire was same as that of their spouses have reduced odds of having unmet http://aps.journals.ac.za need for contraception. This finding is not surprising in that spousal agreement on fertility desire is an indication of such couples being able to discuss their fertility desires. This indicates that such spouses are able to share information as well as their feelings concerning reproductive health issues. Studies elsewhere reveal that women who reported discussion of family planning with spouses have reduced odds of experiencing unmet need for contraception (Wablembo, Notzi and Kwagala 20II; Ajong, Njotang, Yakum et al. 2016). It is therefore most likely that such couples agree on contraceptive use and thus decide to space or limit their births. Actually, the positive relationship between spousal communication and contraceptive use is highlighted by findings of several existing studies such as Sharan and Valente (2002); Lwelamira, Mnyamagola and Msaki (20I2); Islam, Alam and Hasan (20I4); Tilahun, Coene, Temmerman et al. (20I4).

\section{Conclusion}

The study has established that familial factors are to some extent associated with unmet need for contraception among women in Malawi, Togo and Uganda. The fact that women whose fertility desire was the same as their spouses are less likely to have unmet need for contraception underscores the need to come up with strategies which will encourage spousal communication which in turn will aid males and females to be equal partners in family planning decisions.

\section{Acknowledgements}

The authors acknowledge the support of the DSTNRF Centre of Excellence in Human Development, South Africa. Opinions expressed and conclusions arrived at are those of the authors and not to be attributed to the Centre of Excellence in Human Development.

\section{References}

Ahmed, S., Li, Q., Liu, L. and Tsui, A.O. (20I2) "Maternal Deaths Averted by Contraceptive

Use: An analysis of 172 Countries", Lancet, 380 (9837): I I I-I 25.

Ajong, A.B., Njotang, P.N., Yakum, M.N., Essi, M.J., Essiben, F., Eko, F.E, Kenfack, B. and Mbu, E.R. (2016). "Determinants of unmet need for family planning among women in Urban Cameroon: a cross sectional survey in the 
Biyem-Assi Health

BMC Women's

I0.| I 86/s I 2905-0 |6-

District, Yaoundé", Health 16:4, DOI 0283-9

Andi, J.R., Wamala, R., Ocaya, R. and Kabagenyi, A. (20l4). "Modern contraceptive use among women in Uganda: An analysis of trend and patterns (1995-20 I I)", African Population Studies, 28(2): 1009-1021, Doi:10.11564/28-0553.

Audu, B., Yahya, S., Geidama, A., AbduSsalam H., Takai I, and Kyari O. (2008). "Polygamy and the use of contraceptives", International Journal of Gynaecology and Obstetrics, I0I(I): 88-92.

Barman, S. (20/3). "Socio-economic and demographic factors of unmet need for planning in India and its consequences", Research on Humanities and Social Sciences, 3(3), www.iste.org.

Baschieri, A., Cleland, J., Floyd, S., Dube, A., Msona, A., Molesworth, A., Glynn, J.R. and French, N. (20|3). "Reproductive Preferences and Contraceptive Use: A Comparison of monogamous and polygamous couples in Northern Malawi”, Journal of Biosocial Science, 45, 145-166, Doi: 10.1017/S0021932012000569.

Clifton D. and Kaneda T. (2013). "Family Planning Worldwide 2013 Data Sheet",

Washington DC: Population Reference Bureau.

Ejembi C.L., Dahiru, $T$ and Aliyu A.A. (2015).

"Contextual Factors Influencing Modern

Contraceptive Use in Nigeria”. DHS Working Papers No. 120. Maryland, USA: Rockville, ICF International.

Ettarh, R.R. (20I I). Spatial Analysis of Contraceptive Use and Unmet Need in Kenya, Nairobi:

African Population and Health Research Center (APHRC).

Ettarh, R.R and Kyobutungi, C. (20/2). "Physical access to Health Facilities and Contraceptive Use in Kenya: Evidence from the 2008-2009 Kenya Demographic and Health Survey", African Journal of Reproductive Health, 16(3): 48-56.

Hajason, J.Z. Piña, K. and Raveloharimisy, J.L. (20l3). "The Influence of Family Dynamics on Contraceptive Use in Madagascar and the ensuing impact on family well being",

MEASURE

Evaluation PRH, Working Paper Series, Chapel Hill: University of North Carolina.

Horrell S. and Krishnan P. (2007). "Poverty and Productivity in Female-Headed Households in Zimbabwe", Journal of Development Studies, 43(8): I35 I-1380. Doi: 10.1080/ $0022038070161 \mid 47$.

Islam M. A., Padmadas, S. S., and Smith, P. W. F. (2010)."Understanding Family Planning communication in Bangladesh DHS”. GENUS, LXVI

(I), I-I5, http://scistat.cilea.it/index.php/genus/article//down load/76/I I 2 .

Islam, S., Alam, M.S and Hasan, M. (20|4). "InterSpousal Communication on Family

Planning and Its Effect on Contraceptive Use and Method Choice in Bangladesh", Asian Social Science, 10(2): |89-20|.

Kent, M.M (2010). What Would It Cost to Meet Family Planning Needs in Developing

Countries, Meeting Unmet Needs of Family Planning, Population Reference Bureau,

http://www.prb.org/Publications/Articles/2010/ad dingupfpcosts.aspx.

Khan, M.M., Hossain, M.E and Hoq, M.N., (2012). "Determinants of Contraception Use among Female Adolescents in Bangladesh"”,

Asian Social Science, 8(12): |81-191.

Lwelamira J, Mnyamagola G and Msaki M.M. (2012).

"Knowledge, Attitude and Practice

(KAP) towards modern contraceptives among married women of reproductive age

in Mpwapwa District, Central Tanzania”, Current Research Journal of Social Sciences, 4(3):235-245.

Makatjane, T. (1997). "Contraceptive Prevalence in Lesotho: Does the Sex of the Household

Head matter?" African Population Studies, I2(2),http://bioline.org.br/request?ep97010.

Miller, B.C. (2002). Family influences on adolescent sexual and contraceptive behaviour,

Journal of Sex Research, 39: I, 22-26.

Miller, B.C., Benson B. and Galbraith, K.A. (200I). “

Relationships and Adolescent Pregnancy Risk: A

Research Synthesis”, Developmental Review 21, I-38,

Doi: 10.1006/drev.2000.05 I3.

Ms/Togo and ICF International, (2015). Enquête Démographique et de Santé au Togo 20/3-20/4, Rockville,Maryland, USA:

MPDAT, MS and ICF International.

National Statistical Office (NSO) and ICF Macro.

(20I I). Malawi Demographic and Health

Survey 2010, Zomba, Malawi, and Calverton, Maryland, USA: NSO and ICF Macro.

Oginni A.B., Ahonsi, B.A and Adebajo, S. (20I5). "Trend and Determinants of Unmet Need for Family Planning Services among Currently Married Women and Sexually Active Unmarried Women Aged 15-49 in Nigeria (2003-2013)", African Population Studies, 29(I): |483-|499. Osmani A.R., Reyer, J.A, Osmani A.K and Hamajima, N. (20I5). "Factors influencing contraceptive use among women in Afghanistan: secondary analysis of Afghanistan 
Health Survey 2012", Nagoya Journal of Medical Science (77): 55I-56I.

Palamuleni, M.E. (20I3). "Socio-Economic and Demographic Factors Affecting

Contraceptive use in Malawi', African Journal of Reproductive Health, 17(3): 9I- 104.

Peres, C.A., Rutherford, G., Borgesa, G., Galanoa, E., Hudes, E.S., and Hearst, N. (2008). Family

Structure and Adolescent Sexual Behaviour in a Poor Area of São Paulo,

Brazil, Journal of Adolescent Health 42 (2008) I77-I83.

Sahoo H. (2007). "Determinants of contraceptive use in Orissa: An analysis from National Family Health Survey III, Health and Population", Perspectives and Issues 30 (3): 208221.

Sharan, M and Valente, T.W. (2002). "Spousal Communication and Family Planning Adoption: Effects of a Radio Drama Serial in Nepal", International Family Planning Perspectives, 28(I): 16-25.

Sherin R. T., Tiwari, V.K. and Singh, J.V. (20I3). Regional Variations in unmet need for

Family Planning in Rajastan, Health and Population - Perspectives and Issues 36 (l \& 2): 26-44.

Singh, S. \& Darroch, J.E. (20/2). Adding it up: Costs and Benefits of Contraceptive Services Estimates for 2012, New York: Guttamacher Institute and UNFPA.
Stark, L., Tan, T.M., Muldoon, K.A., King, D., Lamin, D.F.M., Lilley, S. and Wessells,

M.G. (20I5). "Family structure and sexual and reproductive health outcomes among adolescents in rural Sierra Leone", Global Public Health, DOI:

I0.1080/I744I692.20I5.I03|I55.

Stover, J. and Ross, J. (2010). "How Increased Contraceptive Use has Reduced Maternal Mortality", Maternal Child Health Journal, 4:687-695, DOI 10.1007/s/0995-0090505-y.

Tilahun T., Coene, G., Temmerman $M$ and Degomme, O. (2014). "Spousal discordance on fertility preference and its effect on contraceptive practice among married couples in Jimma zone, Ethiopia", Reproductive Health II:27, http://www.reproductive- "healthjournal.com/content/I I/I/27.

Uganda Bureau of Statistics (UBOS) and ICF International Inc. (20I2), Uganda

Demographic and Health Survey 201 I, Kampala, Uganda: UBOS and Calverton, Maryland: ICF International Inc.

Wablembo M.S, Notzi J and Kwagala B. (201I). "Does Couple Discussion Influence Unmet Need for Family Planning in Uganda?" Princeton Education Papers, uaps20I I.princeton.edu/papers/I I038I.

All named authors have contributed sufficiently to the work submitted and that the content of the manuscript has never been previously published. 